ARTICLE

\title{
Programming ultrasensitive threshold response through chemomechanical instability
}

Young-Joo Kim (1) 1, Junho Park², Jae Young Lee (D) ${ }^{2}$ \& Do-Nyun Kim (D) 1,2,3凶

The ultrasensitive threshold response is ubiquitous in biochemical systems. In contrast, achieving ultrasensitivity in synthetic molecular structures in a controllable way is challenging. Here, we propose a chemomechanical approach inspired by Michell's instability to realize it. A sudden reconfiguration of topologically constrained rings results when the torsional stress inside reaches a critical value. We use DNA origami to construct molecular rings and then DNA intercalators to induce torsional stress. Michell's instability is achieved successfully when the critical concentration of intercalators is applied. Both the critical point and sensitivity of this ultrasensitive threshold reconfiguration can be controlled by rationally designing the cross-sectional shape and mechanical properties of DNA rings.

\footnotetext{
${ }^{1}$ Institute of Advanced Machines and Design, Seoul National University, Seoul, Korea. ${ }^{2}$ Department of Mechanical Engineering, Seoul National University, Seoul, Korea. ${ }^{3}$ Institute of Engineering Research, Seoul National University, Seoul, Korea. ${ }^{凶}$ email: dnkim@snu.ac.kr
} 
C onstructing an artificial molecular structure with an ultrasensitive threshold response, characterized as a switch-like sigmoidal input-output relationship, has been one of the key challenges in the field of synthetic molecular selfassembly ${ }^{1-9}$. It is ubiquitous in numerous biochemical processes and serves as an essential building block to generate emergent behaviors for cellular regulation ${ }^{10-13}$. Molecular titration is a typical mechanism of ultrasensitivity found in natural molecular networks ${ }^{14-16}$. It occurs when input molecules are sequestered or buffered in inactive complexes by inhibitors, and then an ultrasensitive threshold response emerges after all inhibitors are consumed. The simplicity of molecular titration renders it attractive for encoding ultrasensitivity into synthetic building blocks. However, controlling the trigger point and the sensitivity of a threshold response independently is limited because they are affected by the concentration and affinity of inhibitors ${ }^{16,17}$, but modifying the latter is often challenging.

Here, we propose a chemomechanical mechanism for realizing the ultrasensitive threshold response at the molecular level with highly programmable operating conditions and ranges. We were inspired by Michell's instability ${ }^{18,19}$, which indicates that a topologically constrained ring can be suddenly reconfigured when the torsional stress inside the structure reaches a critical value. We construct molecular rings using the DNA origami method $^{20-22}$, and torsional stress is applied to them by a DNA intercalator. In this study, we employ ethidium bromide (EtBr), doxorubicin (DOX), and dimeric cyanine dye oxazole yellow (YOYO-1) as representative intercalators. Applying critical concentrations of intercalators successfully initiates Michell's instability at the molecular scale. We are able to control the critical point and the sensitivity of this ultrasensitive threshold reconfiguration by rationally designing the cross-sectional shape and the mechanical properties of DNA rings.

\section{Results and discussion}

Chemomechanical instability. We employed a buckling-based structural bifurcation of a ring structure, called Michell's instability (Fig. 1a) ${ }^{18,19}$. The ring structure is formed by joining two ends of a straight rod with a certain amount of twist. Both ends are topologically constrained to prevent their free rotation. The system maintains a planar ring conformation as long as the applied twist $(\theta)$ is below the critical value $\left(\theta_{\text {cr }}\right)$. As shown in the result of finite element (FE) analysis for the ring (Fig. 1b and Supplementary Note 1), the applied twist is stored purely as torsional strain energy in the structure, resulting in zero writhe $\left(\mathrm{W}_{\mathrm{r}}\right)$ and a constant in-plane bending strain energy. However, when it exceeds $\theta_{\mathrm{cr}}$, the ring structure suddenly becomes supercoiled with a nonzero writhe due to Michell's instability, where the stored torsional strain energy is drastically transformed into the out-of-plane bending energy. Hence, rings are capable of buffering a certain amount of torsional strain energy, enabling a switch-like, threshold reconfiguration from a circle to a supercoil under varied twist angles.

To investigate Michell's instability at the molecular level, we constructed ring structures using the DNA origami method ${ }^{20-22}$ (Fig. 1c). Various cross-sections and curvatures can be easily programmed by designing the sequences of short single-stranded DNAs that fold a long, viral single strand into a ring. We carefully designed the structures to minimize unwanted torsional prestress due to the interplay between bending and torsion ${ }^{21}$. DNA intercalators were employed as chemomechanical stimuli to induce torsion in the ring. They are known to perturb the canonical geometry of B-form double-stranded DNA (dsDNA), especially lowering the twist angle between neighboring base pairs (BPs) by 23, 24, and 26 degrees for DOX, YOYO-1, and EtBr, respectively ${ }^{23-25}$. Since neighboring DNA helices are crosslinked, the geometrical perturbation of dsDNA caused by their binding induces torsional stress in the ring structure, eventually resulting in positive (i.e., left-handed) supercoiling when it reaches its critical value (Fig. 1d). Hence, DNA origami rings exhibit a steep conformational change due to chemomechanically driven Michell's instability. Note that straight DNA origami structures, unlike topologically constrained rings, would be twisted gradually along the helical axis to relieve torsional stress without buckling in response to intercalators ${ }^{26-28}$.

Ultrasensitive threshold reconfiguration. To demonstrate this, we built six-helix-bundle (6HB) and ten-helix-bundle (10HB) DNA origami rings with and without topological constraints (Fig. 2a). Open ring structures showed wide distributions in the radii of curvature, which corresponded to $90.7 \pm 11.4$ and $51.1 \pm 6.9 \mathrm{~nm}$ for $6 \mathrm{HB}$ and $10 \mathrm{HB}$, respectively. Topologically constrained, closed rings were formed by connecting both ends of open rings with additional DNA single strands, resulting in much narrower distributions in the radii of curvature, which were measured as $61.7 \pm 0.9$ and $35.1 \pm 1.4 \mathrm{~nm}$ for $6 \mathrm{HB}$ and $10 \mathrm{HB}$, respectively. For all structures, high folding yields of well-folded monomers were achieved (Supplementary Fig. 1).

We first used atomic force microscopy (AFM) images to analyze the conformational changes of these ring structures in response to systematically varied concentrations of $\mathrm{EtBr}$ in a model system, since $\mathrm{EtBr}$ is a mono-intercalator inducing the highest unwinding to DNA among the three intercalators employed in this study. The binding density of EtBr for DNA nanostructures was $\sim 0-0.2$ molecules per $\mathrm{BP}$ in the range of concentrations we investigated ${ }^{27}$. We measured the ratio of noncircular (coiled or twisted) structures $\left(\mathrm{R}_{\mathrm{NC}}\right)$ among wellfolded monomers (Fig. 2b and Supplementary Figs. 2-19). For open rings, $\mathrm{R}_{\mathrm{NC}}$ gradually increased with $\mathrm{EtBr}$ concentration. Because both ends could be freely rotated, they became lefthanded helical coils with a gradually increasing twist rate as the concentration of $\mathrm{EtBr}$ increased (Fig. 2c). In contrast, ultrasensitive threshold responses were clearly seen in the $\mathrm{R}_{\mathrm{NC}}$ curves for closed rings. Almost no change in $\mathrm{R}_{\mathrm{NC}}$ was observed for $\mathrm{EtBr}$ concentrations below the critical concentrations, which were $1 \mu \mathrm{M}$ for $6 \mathrm{HB}$ and $4 \mu \mathrm{M}$ for $10 \mathrm{HB}$, and then, $\mathrm{R}_{\mathrm{NC}}$ spiked and quick saturation occurred. At these critical concentrations, $\sim 43-58 \%$ of monomers were suddenly transformed into supercoiled conformations. The effective Hill coefficient $\left(\mathrm{N}_{\mathrm{H}}\right)$ was estimated by fitting $\mathrm{R}_{\mathrm{NC}}$ curves to the Hill function to quantify the sensitivity of the threshold response ${ }^{29,30}$. Closed $6 \mathrm{HB}$ and $10 \mathrm{HB}$ rings showed coefficient values of 11.1 and 10.3 , respectively, representing high levels of ultrasensitivity upon $\mathrm{EtBr}$ binding ${ }^{12,31}$, while open rings exhibited low values of 1.8 and 2.4, respectively. Similar trends were observed using the radius of gyration $\left(\mathrm{R}_{\mathrm{g}}\right)$ of ring structures at various $\mathrm{EtBr}$ concentrations (Supplementary Fig. 20).

While $6 \mathrm{HB}$ and $10 \mathrm{HB}$ closed rings showed similar levels of ultrasensitivity, their critical concentrations for triggering the buckling transition were quite different. This primarily arose from differences in their mechanical properties, particularly in the ratio of bending rigidity (B) to torsional rigidity (C). A closed ring with a higher $\mathrm{B} / \mathrm{C}$ ratio would require a higher torsional stress to initiate buckling ${ }^{18,19}$. Generally, the bending rigidity is known to be proportional to $\mathrm{N}^{2}$, where $\mathrm{N}$ is the number of helices in the bundle, while the torsional rigidity increases linearly with respect to $\mathrm{N}^{32-34}$. Accordingly, the $\mathrm{B} / \mathrm{C}$ ratio is approximately proportional to $\mathrm{N}$, as was also confirmed computationally by normal mode analysis (NMA) (Fig. 2e and Supplementary Note 3). Hence, the critical concentration of $\mathrm{EtBr}$ for the $10 \mathrm{HB}$ closed ring 
a

(i)<smiles>c1ccc2ccccc2c#1</smiles>

$=$
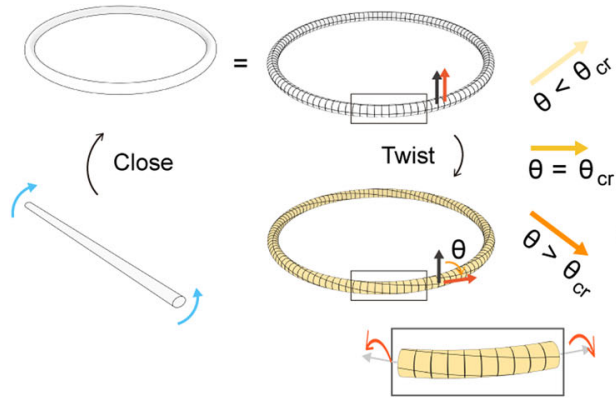

\section{(1)}

(in)

(1)

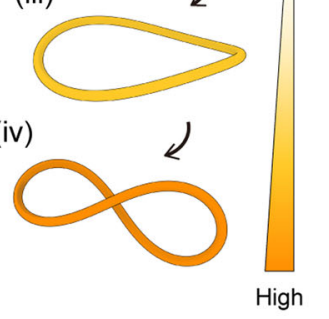

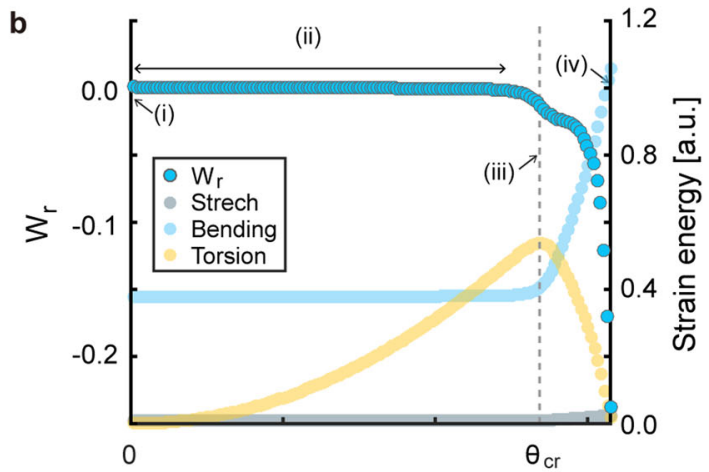

Twist $(\theta)$

C



d
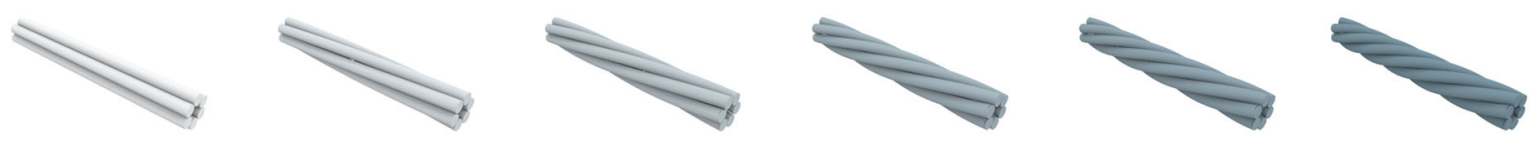

Intercalator concentration
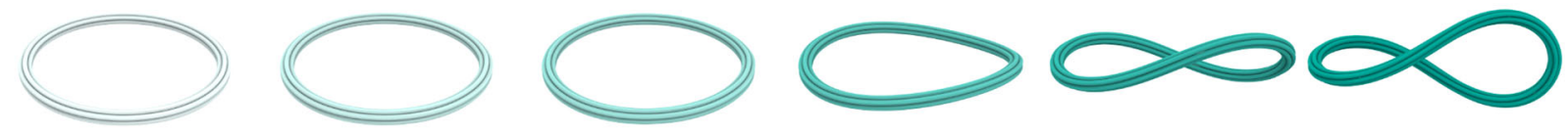

Fig. 1 Chemomechanical instability of DNA rings. a Michell's instability. A ring structure is formed by joining both ends of a straight rod with a certain amount of twist. Color bar represents the applied twist angle $(\theta) . \theta_{c r}$ denotes the critical angle triggering the instability. $\mathbf{b} W_{r i t h e}\left(W_{r}\right)$ and the strain energy of the ring as a function of the applied twist angle. $\mathbf{c}$ Realizing Michell's instability with DNA rings. Intercalators perturb the geometry of DNA when bound and induce torsional stress on the ring. $\mathbf{d}$ A topologically unconstrained straight bundle (top) shows a gradual reconfiguration with respect to the concentration of an intercalator, while an ultrasensitive threshold reconfiguration occurs for a topologically constrained ring structure (bottom).

would be higher than that for the $6 \mathrm{HB}$ closed ring. This result shows the great flexibility of the proposed method for controlling the threshold response. We are able to tailor the critical concentration for the buckling transition through the structural design of DNA bundle rings while maintaining the level of ultrasensitivity, which has been well established in structural DNA nanotechnology ${ }^{21,22}$.

Moreover, the proposed ultrasensitive reconfiguration was reversible, suggesting that it was mostly based on elastic deformation. $\mathrm{R}_{\mathrm{NC}}$ of the $6 \mathrm{HB}$ ring was measured after adding $2 \mu \mathrm{M} \mathrm{EtBr}$ and removing it by buffer exchange (Fig. 2f and Supplementary Fig. 21) ${ }^{35}$. The results showed that even one buffer exchange was enough to return the supercoiled structure back to its planar conformation, and the reconfiguration was repeatable.

Other intercalators can be employed as chemomechanical stimuli. As each of them has different binding affinities and induces different geometrical perturbations in DNA, the trigger point and the sensitivity of the threshold response to it are expected to be different. We tested DOX, a mono-intercalator often used as an anticancer drug, and YOYO-1, a bis-intercalator widely used for green fluorescence (Fig. 2g and Supplementary Figs. 22-27). Ultrasensitivity was clearly observed for these agents, with a Hill coefficient of 9.2 for both DOX and YOYO-1. The response curve for DOX was similar to that for $\mathrm{EtBr}$, probably because they have the same intercalation mode (monointercalator) and similar binding affinity to $\mathrm{DNA}^{36,37}$. On the other hand, YOYO-1 generated the buckling transition at a concentration that was an order of magnitude lower than those of $\mathrm{EtBr}$ and DOX. Its distinct intercalation mode (bis-intercalation) and higher binding affinity to DNA ${ }^{25,36,37}$ might explain this result, since all three agents effect unwinding of DNA to a similar extent after binding.

Sensitivity modulation. The driving force for the reconfiguration of DNA origami rings is chemomechanical (torsional) stress arising from geometrical perturbation in DNA duplexes induced by intercalators. Recently, it was shown that mechanical stress in DNA bundles could be relaxed systematically by introducing short single-stranded regions called gaps into the structure ${ }^{38}$. 
a

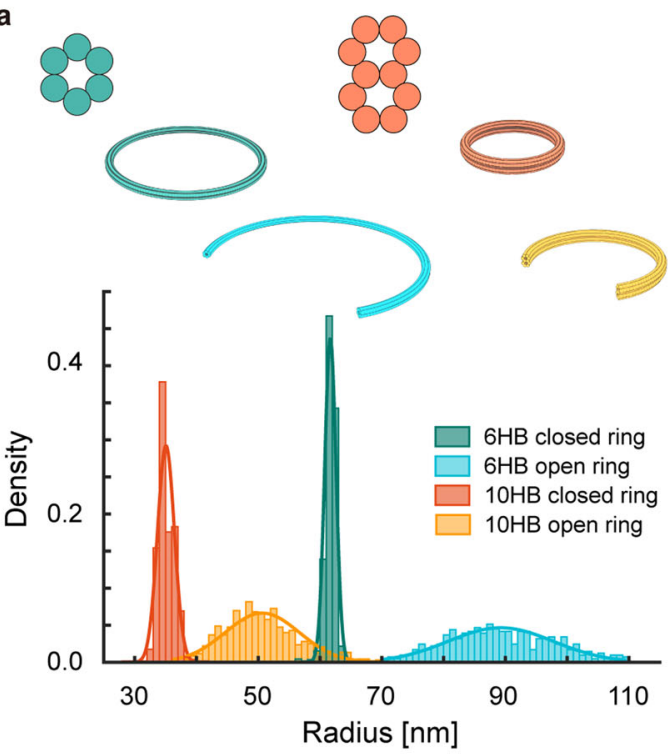

b

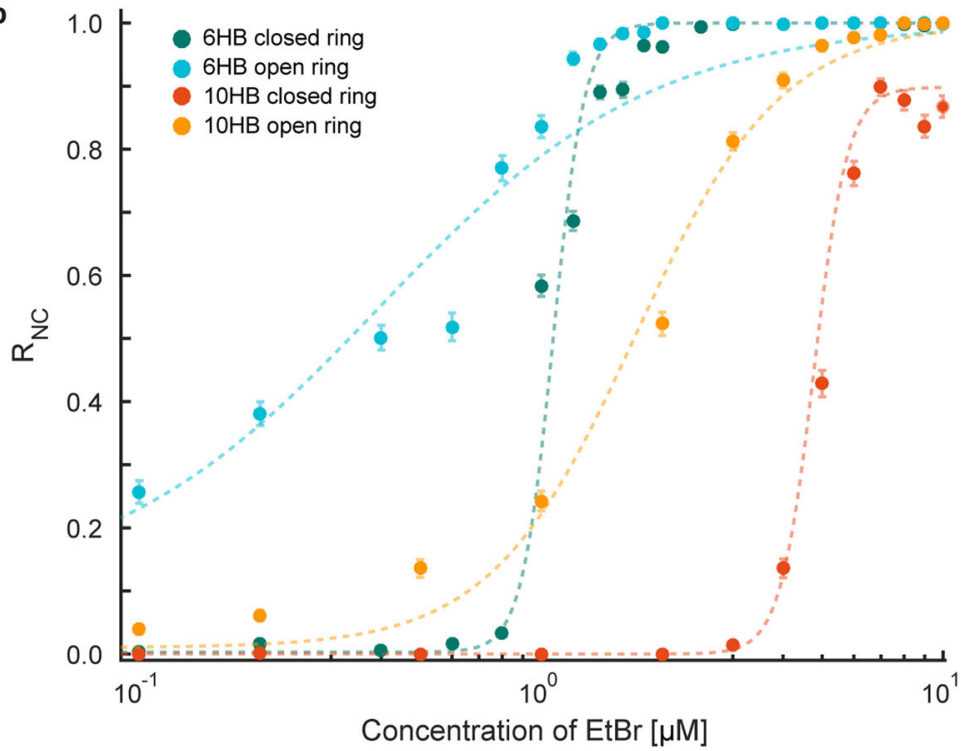

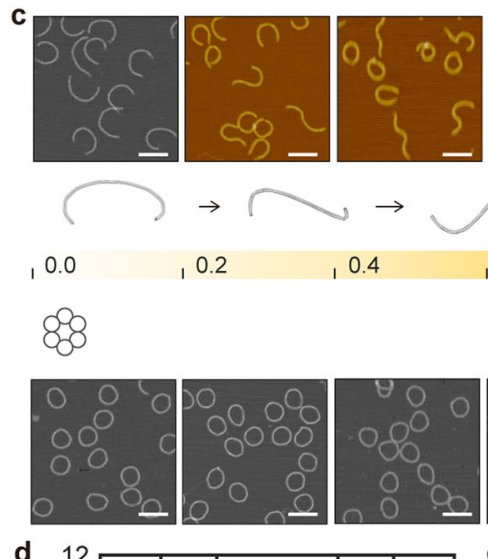
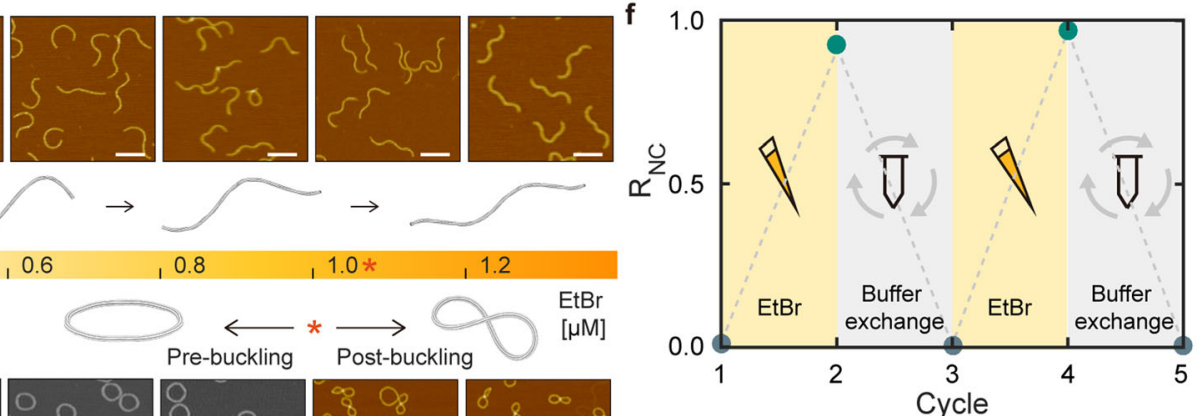

0.6
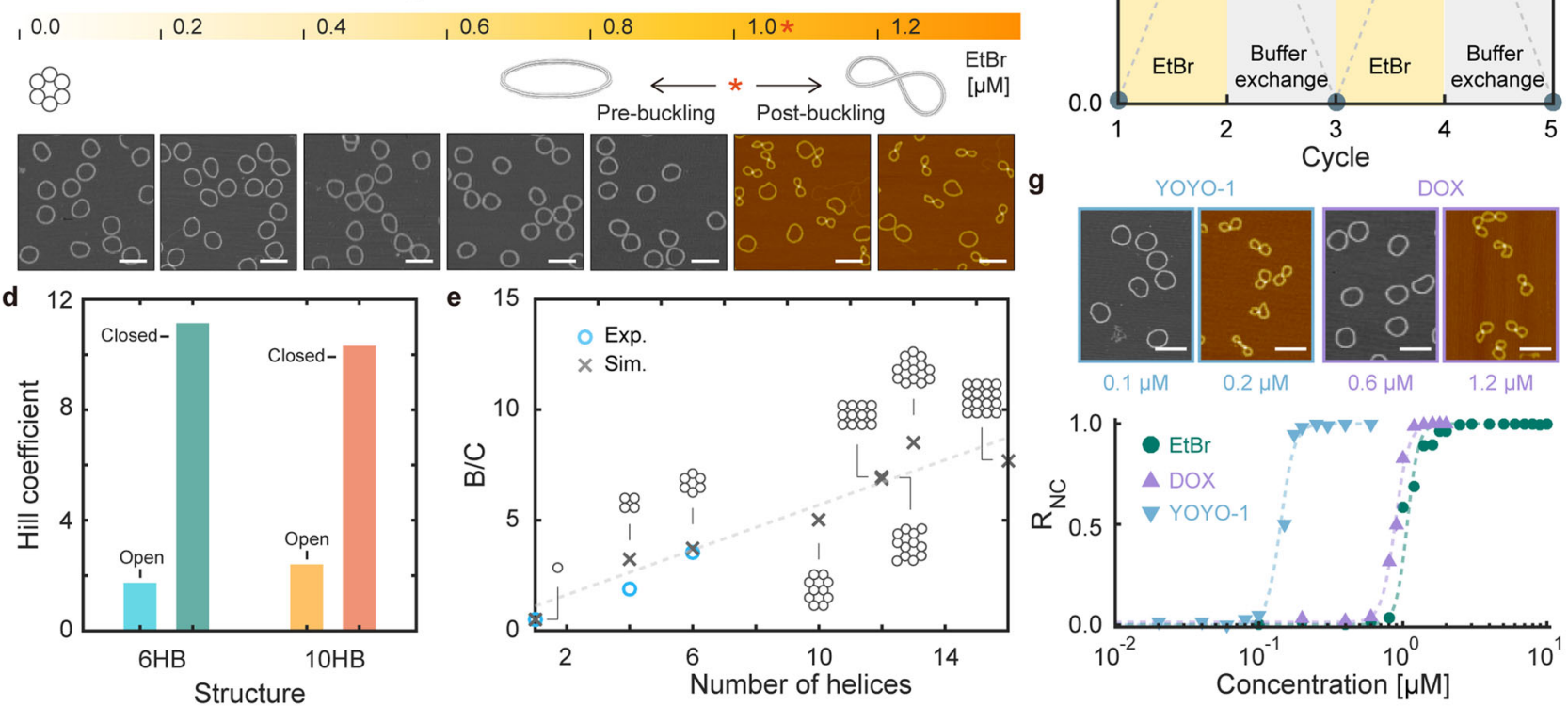

Fig. 2 Reconfiguration of open and closed DNA rings. a Schematics and histograms for experimentally measured radii of curvature for $6 \mathrm{HB}$ and $10 \mathrm{HB}$ rings. $\mathbf{b}$ Ratio of noncircular monomers $\left(R_{N C}\right)$ as a function of EtBr concentration. Circles and error bars represent the mean and the standard deviation of experimentally measured $R_{N C}$ values, respectively. Dashed lines represent the fitted Hill curves. c Representative AFM images and predicted configurations (Supplementary Note 2) of 6HB open (top) and closed (bottom) rings. Scale bars in the AFM images represent $200 \mathrm{~nm}$. d Effective Hill coefficients. e Ratio of bending rigidity to torsional rigidity $(B / C)$ of DNA bundles with various cross-sectional shapes. Blue circles and black crosses represent experimental ${ }^{32,55}$ and predicted values, respectively. The dashed line shows a linear fit to the predicted B/C values. $\mathbf{f}$ Reversibility of ultrasensitive reconfiguration. $\mathbf{g}$ Ultrasensitive threshold reconfiguration of $6 \mathrm{HB}$ closed rings by DOX and YOYO-1. Markers and error bars represent the mean and the standard deviation of experimentally measured $R_{N C}$ values, respectively. Dashed lines represent the fitted Hill curves. Scale bars in the AFM images represent $200 \mathrm{~nm}$.

Since the sensitivity of reconfiguration is governed by the level of torsional stress induced by intercalators, we might be able to control it by rationally designing the location and proportion of these gaps in the structure.
To explore this hypothesis, we constructed $6 \mathrm{HB}$ rings by placing gaps at nick (single-strand break) positions (Fig. 3a). To minimize undesired structural changes, nicks were placed on helices without any geometrical perturbation and were replaced 
a
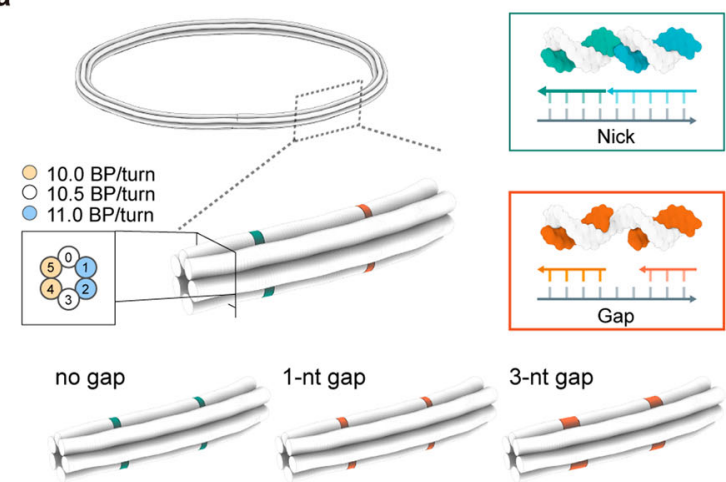

b
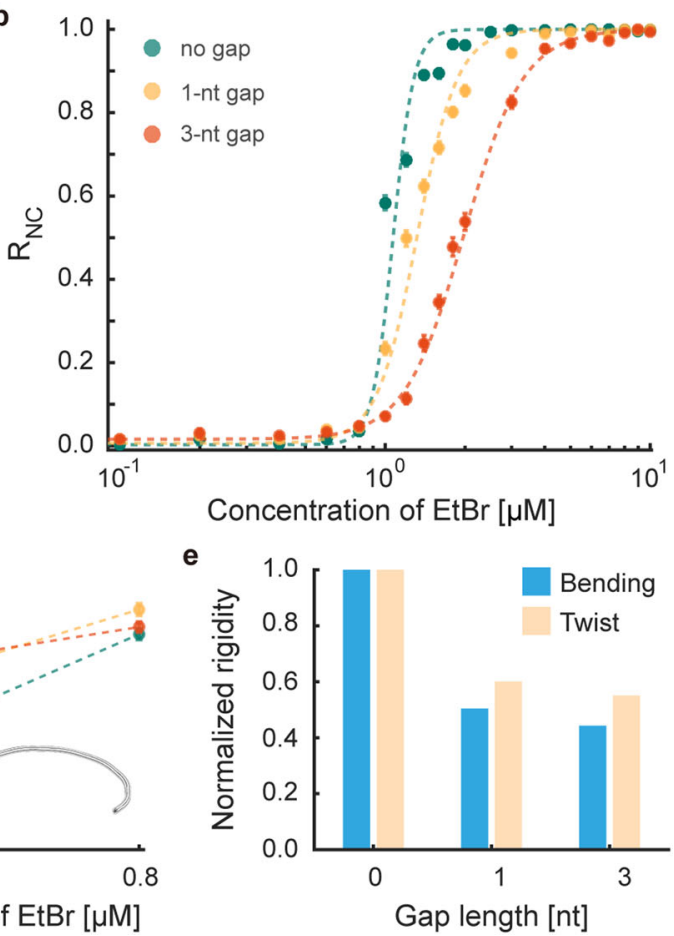

Fig. 3 Sensitivity modulation through stress relaxation by gaps. a $6 \mathrm{HB}$ closed rings with gaps. b $\mathrm{R}_{\mathrm{NC}}$ values as a function of EtBr concentration. Circles and error bars represent the means and the standard deviations of experimentally measured $R_{N C}$ values, respectively. Dashed lines represent the fitted Hill curves. c Effect of the gap length on the effective Hill coefficient. $\mathbf{d}$ Effect of gaps on the configurational change of $6 \mathrm{HB}$ open rings. e Effect of gaps on the mechanical properties of straight $6 \mathrm{HB}$ structures.

with gaps only when the crossover spacing was 14 BPs long to guarantee sufficient binding energies of staple strands at the gap positions necessary for high folding yield ${ }^{39,40}$. The level of stress relaxation was adjusted by the gap length. We used onenucleotide (1-nt) and three-nucleotide (3-nt) gaps, which did not deteriorate the structural integrity of $6 \mathrm{HB}$ rings (Supplementary Fig. 28). Analyses of AFM images for these structures at various $\mathrm{EtBr}$ concentrations revealed that gaps lowered the sensitivity of reconfiguration while maintaining its threshold response (Fig. 3b and Supplementary Figs. 29-38). Hill coefficients were reduced from 11.1 to 5.6 and 3.9 when 1-nt and 3-nt gaps were used, respectively (Fig. 3c). As we can vary the length, location, and number of gaps broadly 38,40 , the level of stress relaxation and hence the sensitivity of reconfiguration can be controlled more widely and finely.

Note that the critical concentration of EtBr triggering the buckling transition was not changed much by gaps, unlike the sensitivity of reconfiguration. This was counterintuitive because stress relaxation by gaps would require a higher concentration of $\mathrm{EtBr}$ to induce the torsional stress required for initiating Michell's instability. Hence, there must be other effects of gaps on the structure that nullify the effect of stress relaxation on the critical point. We found two potential explanations for the minimal change in the critical concentration of EtBr caused by gaps. First, we investigated the conformational changes of $6 \mathrm{HB}$ open rings with and without gaps with respect to EtBr concentration. Unlike closed rings with topological constraints, large portions of open rings with gaps (30 to $55 \%$ ) were folded into nonplanar, helical coils with left-handedness even without the presence of EtBr (Fig. 3d and Supplementary Figs. 39 and 40). The portion of helical coils was higher when gaps were used and monotonically increased with increasing EtBr concentration. This suggests that the left-handed twist was more readily induced by gaps in the open ring and gaps created torsional prestress when topologically constrained into closed planar rings; this lowered the chemomechanically applied critical twist required for the buckling transition. Second, gaps affect the mechanical properties of DNA bundles. Normal mode analysis using FE models for straight $6 \mathrm{HBs}$ with and without gaps revealed that both torsional rigidity (C) and bending rigidity (B) were decreased by gaps (Fig. 3e and Supplementary Note 3$)^{40}$. In particular, the $\mathrm{B} / \mathrm{C}$ ratio decreased with gaps as the reduction rate of $\mathrm{B}$ was greater than that of $\mathrm{C}$. As a lower $\mathrm{B} / \mathrm{C}$ ratio requires a lower torsional stress for Michell's instability, it lowers the required critical concentration of $\mathrm{EtBr}^{18,19}$. These two structural effects caused by gaps (generation of torsional prestress and reduction of the $\mathrm{B} / \mathrm{C}$ ratio) might compensate for the effect of stress relaxation, resulting in barely noticeable differences in the critical concentration. In fact, this offers a versatile way of controlling the threshold response via structural design. We may independently modulate the critical concentration of chemomechanical stimuli by using crosssectional design of rings and the sensitivity by gaps or engineered defects $^{40}$.

Importance of shape homogeneity. When we design an ultrasensitive threshold response based on Michell's instability, both the topological constraint forming a closed structure and the homogeneity of the structural shape in the ring are important. To illustrate, we designed a $6 \mathrm{HB}$ closed triangle using the same crosssectional shape and the bundle length of the $6 \mathrm{HB}$ closed ring (Supplementary Fig. 41). The triangle has straight edges and curved vertices, and hence, the deformation energy is localized near the vertices in contrast to the ring, where it is rather homogeneously distributed throughout the structure. The $6 \mathrm{HB}$ closed triangle showed a gradual reconfiguration upon $\mathrm{EtBr}$ binding similar to those of open rings, but without a threshold (Fig. 4a and Supplementary Figs. 42-56). Local coiling modes 




c



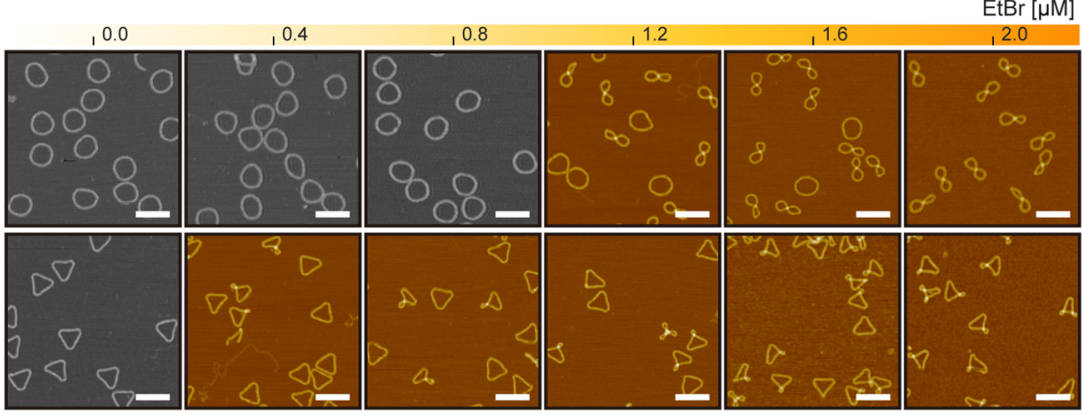

d

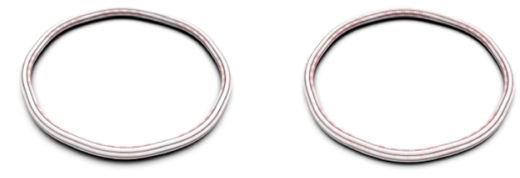

1.1
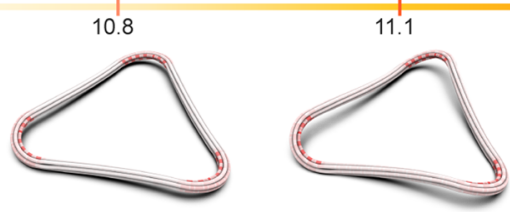


Fig. 4 Differences in the reconfiguration responses between rings and triangles. a $R_{N C}$ values as a function of EtBr concentration. Circles and error bars represent the means and the standard deviations of experimentally measured $R_{N C}$ values, respectively. Dashed lines represent the fitted Hill curves. $\mathbf{b}$ AFM images of $6 \mathrm{HB}$ closed rings (top) and triangles (bottom). Scale bars in the AFM images represent $200 \mathrm{~nm}$. c Root-mean-squared displacement (RMSD) of $6 \mathrm{HB}$ closed rings (green) and triangles (red) predicted by FE analysis. RMSD was calculated for structures with perturbed helicities with respect to the reference structure exhibiting a helicity of $10.5 \mathrm{BP} /$ turn. $\mathbf{d}$ Simulated conformations of $6 \mathrm{HB}$ closed rings (top) and triangles (bottom). The color bar represents the strain energy stored in BPs.

emerged at the vertices even at low concentrations of EtBr without any buffering of induced torsional strain energy (Fig. 4b).

FE analysis for closed ring and triangle structures captured these behaviors as well. Their three-dimensional shapes and strain energies were calculated by varying the helicity of dsDNA to consider the effect of EtBr binding (Supplementary Note 2). The root-mean-square-displacement (RMSD) curves showed a remarkable difference between the two structures in terms of conformational changes with respect to helicity (Fig. 4c). The closed ring maintained its circular shape in a plane with no significant conformational change, and its strain energy almost uniformly increased as the torsional energy throughout the structure increased (Fig. 4d). In contrast, the closed triangle was gradually reconfigured by changes in helicity, with local coiling occurring near its vertices where much higher strain energy was concentrated. The triangular structure could not maintain a planar configuration with distinct deformation energies induced at vertices and edges while still satisfying geometrical compatibility under the topological constraint. Therefore, it would be essential to design a structure with shape homogeneity so that chemomechanically induced deformation energies could be distributed uniformly throughout the structure to achieve an ultrasensitive threshold response.

We showed that ultrasensitive threshold responses could be programmed into synthetic molecular structures via chemomechanical instability without inhibitor molecules. Due to Michell's instability, topologically constrained molecular rings were capable of buffering chemomechanically induced stresses without conformational change. While Michell's instability was used to explain the supercoiling of dsDNA rings theoretically ${ }^{41}$, herein, we apply it to structured DNA assemblies in a highly programmable manner. The trigger point and the sensitivity of reconfiguration could be easily modulated by controlling the global and local mechanical properties of rings for a given intercalator.

The proposed mechanism might be realized with other DNA structuring methods, such as DNA tile ${ }^{42}$ and brick methods ${ }^{43}$, and it could also be applicable to other engineered molecular selfassemblies of $\mathrm{RNA}^{44,45}$ or proteins ${ }^{46,47}$. While we used intercalators as a chemomechanical stimuli in this study, any other DNA binding molecules that perturb the geometry of dsDNA, including DAPI, topotecan, netropsin, and cisplatin, can be used alternatively to induce chemomechanical instability $27,37,48$. The use of multiple chemical agents with different binding affinities and structural effects on DNA would broaden the tunable range of ultrasensitive threshold responses even further. Environmental factors, which can affect the binding affinity of chemomechanical stimulus agents, such as salt concentration or $\mathrm{pH}$, can be adopted as additional tuning methods ${ }^{49}$.

Our reconfiguration mechanism would be further utilized to realize ultrasensitivity in other chemical or physical responses by precisely arranging functional or interacting molecules onto DNA nanostructures and using the proximity-induced reactions between them after reconfiguration ${ }^{50-52}$. In addition, DNA rings might be embedded in soft materials such as DNA hydrogels, where they could serve as crosslinkers for scaffold polymers to enable ultrasensitive threshold deformations cause by stimuli.

\section{Methods}

Self-assembly of DNA origami structures. Using an open source program, $\mathrm{caDNAno}^{53}$, all structures used here were designed on the honeycomb lattice with a M13mp18 scaffold strand (7249-nt-long, GUILD, www.guildbioscience.com). Sequences of staple strands for the structures were exported from the caDNAno (Supplementary Tables 1-5) and they were synthesized from Bioneer (www.bioneer.co.kr). A folding mixture consists of $20 \mathrm{nM}$ concentration of scaffold DNA, $100 \mathrm{nM}$ concentration of each staple strand, $1 \times$ TAE buffer $(40 \mathrm{mM}$ Trisacetate and $1 \mathrm{mM}$ EDTA, Bioneer) and $20 \mathrm{mM}$ of $\mathrm{MgCl}_{2}$ (Sigma-Aldrich, 
www.sigmaaldrich.com). The annealing process for self-assembly of DNA strands was performed by establishing temperature gradients from 80 to $60^{\circ} \mathrm{C}$ with a rate of $-0.25^{\circ} \mathrm{C} / \mathrm{min}$ and from 60 to $45^{\circ} \mathrm{C}$ at a rate of $-1{ }^{\circ} \mathrm{C} / \mathrm{hr}$ in a thermocycler (T100, Bio-Rad, www.bio-rad.com). Excessive staple strands were removed through five buffer exchange procedure ${ }^{35}$ at $5 \mathrm{krcf}$ during $8 \mathrm{~min}$ and concentration of structures was adjusted using the same buffer used in folding $(1 \times \mathrm{TAE}$ and $20 \mathrm{mM}$ of $\mathrm{MgCl}_{2}$ ). Concentrations of folded structures were measured using a Nanodrop One UV spectrophotometer (Thermo Fisher Scientific, www.thermofisher.com). Purified structures were stored at $-4{ }^{\circ} \mathrm{C}$ in a refrigerator.

Agarose gel electrophoresis. Annealed samples of DNA origami structures were electrophoresed using $1 \%$ agarose gel containing $0.5 x$ TBE ( $45 \mathrm{mM}$ Tris-borate and $1 \mathrm{mM}$ EDTA, Sigma-Aldrich), $12 \mathrm{mM} \mathrm{MgCl}$, and $0.5 \mu \mathrm{l} / \mathrm{ml} \mathrm{EtBr} \mathrm{(Noble} \mathrm{Bioscience}$ Inc.). Electrophoresis was performed for $120 \mathrm{~min}$ at $60 \mathrm{~V}$ bias voltage in an icewater cooled chamber. Gel imaging was performed using GelDoc XR + device and Image Lab v5.1 program (Bio-Rad).

AFM imaging. Before deposition, the purified sample was diluted by the folding buffer solution and mixed with varying amount of intercalators. $0.4 \mathrm{nM}$ annealed structures were used in order to ensure appropriate numbers of monomers on the substrate for image analysis. The $20 \mu \mathrm{l}$ of diluted sample was then deposited and incubated on a freshly cleaved mica substrate (highest grade V1 AFM Mica, TedPella Inc.) for 5-10 min. The substrate was washed with DI water and gently dried using a $\mathrm{N}_{2}$ gun $\left(<0.1 \mathrm{kgf} / \mathrm{cm}^{2}\right)$. If the number of monomers in images was small, the sample was incubated for a longer time. AFM images were taken by a NX10 system (Park Systems, www.parksystems.co.kr) using the noncontact mode in SmartScan software. A PPP-NCHR probe with spring constant of $42 \mathrm{~N} / \mathrm{m}$ was used in the measurements (Nanosensors). Each image had a sample area of $5 \mu \mathrm{m} \times 5 \mu \mathrm{m}$ in $1024 \times 1024$ pixel resolution. Generally, five to eight AFM images were obtained for one sample for each case to obtain statistically sufficient numbers of monomer particles (at least 300 particles). Images were flattened with linear and quadratic order using the XEI 4.1.0 program (Park Systems). After removing aggregates through filtering by the pixel size, monomeric particle images of DNA origami structures were extracted from AFM images using custom scripts developed in MATLAB R2016a (MathWorks Inc.) and provided in the literature ${ }^{54}$, and classification of single particles was done manually. Only well-folded monomers were used in analyses. Conformations of monomers were manually classified depending on whether they were circular or not. After binarization of collected monomer particles, pixels were fitted to a circle in order to calculate the radius of curvature using custom scripts developed in MATLAB R2016a.

Effective Hill coefficient. Sensitivity of reconfiguration of DNA origami nanostructures was analyzed by fitting the Hill equation, expressed as

$$
\mathrm{R}_{\mathrm{NC}}=\mathrm{R}_{\mathrm{NC}, \text { min }}+\left(\mathrm{R}_{\mathrm{NC}, \text { max }}-\mathrm{R}_{\mathrm{NC}, \text { min }}\right) \frac{\mathrm{x}^{\mathrm{n}_{\mathrm{H}}}}{\mathrm{x}_{50}^{\mathrm{n}_{\mathrm{H}}}+\mathrm{x}^{\mathrm{n}_{\mathrm{H}}}}
$$

where $\mathrm{x}$ is the concentration of intercalator ${ }^{30}$. Here, $\mathrm{R}_{\mathrm{NC} \text {,min }}$ and $\mathrm{R}_{\mathrm{NC} \text {,max }}$ are the minimum and the maximum $\mathrm{R}_{\mathrm{NC}}$, respectively, while $\mathrm{x}_{50}$ refers to the concentration of intercalator required to reach half-maximal $\mathrm{R}_{\mathrm{NC}}$. The equation was fitted by minimization of the sum of squared relative residuals using the function 'fmincon' in MATLAB R2016a.

\section{Data availability}

Data supporting the findings of this study are available in the main manuscript and the supporting information. AFM images not included in the supporting information but used in analyses are available from the authors upon reasonable request.

Received: 21 March 2021; Accepted: 3 August 2021;

Published online: 30 August 2021

\section{References}

1. Gardner, T. S., Cantor, C. R. \& Collins, J. J. Construction of a genetic toggle switch in Escherichia coli. Nat. Chem. 403, 339-342 (2000).

2. Hooshangi, S., Thiberge, S. \& Weiss, R. Ultrasensitivity and noise propagation in a synthetic transcriptional cascade. Proc. Natl Acad. Sci. USA. 102, 3581-3586 (2005).

3. Kim, J., White, K. S. \& Winfree, E. Construction of an in vitro bistable circuit from synthetic transcriptional switches. Mol. Syst. Biol. 2, 68 (2006).

4. Dueber, J. E., Mirsky, E. A. \& Lim, W. A. Engineering synthetic signaling proteins with ultrasensitive input/output control. Nat. Biotechnol. 25, 660-662 (2007),

5. Bashor, C. J., Helman, N. C., Yan, S. \& Lim, W. A. Using engineered scaffold interactions to reshape MAP kinase pathway signaling dynamics. Science 319, 1539-1543 (2008).
6. Vallée-Bélisle, A., Ricci, F. \& Plaxco, K. W. Engineering biosensors with extended, narrowed, or arbitrarily edited dynamic range. J. Am. Chem. Soc. 134, 2876-2879 (2012).

7. Chen, D. \& Arkin, A. P. Sequestration-based bistability enables tuning of the switching boundaries and design of a latch. Mol. Syst. Biol. 8, 620 (2012).

8. Kuzyk, A. et al. Selective control of reconfigurable chiral plasmonic metamolecules. Science Advances 3, e1602803 (2017).

9. Ijäs, H. et al. Reconfigurable DNA Origami Nanocapsule for pH-Controlled Encapsulation and Display of Cargo. ACS Nano 13, 5959-5967 (2019).

10. Koshland, D. E., Goldbeter, A. \& Stock, J. B. Amplification and adaptation in regulatory and sensory systems. Science 127, 220-225 (1982).

11. Bhalla, U. S. \& Iyengar, R. Emergent properties of networks of biological signaling pathways. Science 283, 381-387 (1999).

12. Zhang, Q., Bhattacharya, S. \& Andersen, M. E. Ultrasensitive response motifs basic amplifiers in molecular signalling networks. Open Biol. 3, 130031 (2013).

13. Ferrell, J. E. Jr \& Ha, S. H. Ultrasensitivity part III: cascades, bistable switches, and oscillators. Trends biochemical Sci. 39, 612-618 (2014).

14. Sheridan, J. P. et al. Control of TRAIL-induced apoptosis by a family of signaling and decoy receptors. Science 277, 818-821 (1997).

15. Klein, D. E. et al. Argos inhibits epidermal growth factor receptor signalling by ligand sequestration. Nat. Chem. 430, 1040-1044 (2004).

16. Buchler, N. E. \& Louis, M. Molecular titration and ultrasensitivity in regulatory networks. J. Mol. Biol. 384, 1106-1119 (2008).

17. Ricci, F., Vallée-Bélisle, A., \& Plaxco, K. W. High-precision, in vitro validation of the sequestration mechanism for generating ultrasensitive dose-response curves in regulatory networks. PLoS Comput Biol. 7, e1002171 (2011).

18. Michell, J. On the stability of a bent and twisted wire. Messenger Math. 11, 181-184 (1889).

19. Goriely, A. Twisted elastic rings and the rediscoveries of Michell's instability. J. Elast. 84, 281-299 (2006).

20. Rothemund, P. W. K. Folding DNA to create nanoscale shapes and patterns. Nature 440, 297-302 (2006)

21. Dietz, H., Douglas, S. M. \& Shih, W. M. Folding, DNA into twisted and curved nanoscale shapes. Science 325, 725-730 (2009).

22. Douglas, S. M. et al. Self-assembly of DNA into nanoscale three-dimensional shapes. Nature 459, 414-418 (2009).

23. Wang, J. The degree of unwinding of the DNA helix by ethidium: I. titration of twisted PM2 DNA molecules in alkaline cesium chloride density gradients. J. Mol. Biol. 89, 783-801 (1974).

24. Coury, J. E., McFail-Isom, L., Williams, L. D. \& Bottomley, L. A. A novel assay for drug-DNA binding mode, affinity, and exclusion number: scanning force microscopy. Proceedings of the National Academy of Sciences of the United States of America (PNAS) 93, 12283-12286 (1996).

25. Günther, K., Mertig, M. \& Seidel, R. Mechanical and structural properties of YOYO-1 complexed DNA. Nucleic Acids Res. 38, 6526-6532 (2010).

26. Zhao, Y.-X. et al. DNA origami delivery system for cancer therapy with tunable release properties. ACS Nano 6, 8684-8691 (2012).

27. Chen, H. et al. Dynamic and progressive control of DNA origami conformation by modulating DNA helicity with chemical adducts. ACS Nano 10, 4989-4996 (2016)

28. Zadegan, R. M. et al. Twisting of dna origami from intercalators. Sci. Rep. 7, 7382 (2017).

29. Hill, A. V. The possible effects of the aggregation of the molecules of haemoglobin on its dissociation curves. j. physiol. 40, 4-7 (1910).

30. Legewie, S., Blüthgen, N. \& Herzel, H. Quantitative analysis of ultrasensitive responses. FEBS J. 272, 4071-4079 (2005).

31. Ferrell, J. E. Jr \& Ha, S. H. J. T. I. B. S. Ultrasensitivity part I: Michaelian responses and zero-order ultrasensitivity. Trends Biochem. Sci. 39, 496-503 (2014).

32. Kauert, D. J., Kurth, T., Liedl, T. \& Seidel, R. Direct mechanical measurements reveal the material properties of three-dimensional DNA origami. Nano Lett. 11, 5558-5563 (2011).

33. Castro, C. E. et al. Mechanical design of DNA nanostructures. Nanoscale 7, 5913-5921 (2015)

34. Lee, J. Y. et al. Rapid computational analysis of DNA origami assemblies at near-atomic resolution. ACS Nano 15, 1002-1015 (2021).

35. Wagenbauer, K. F. et al. How we make DNA origami. ChemBioChem 18, 1873-1885 (2017)

36. Schneider, Y. J., Baurain, R., Zenebergh, A. \& Trouet, A. DNA-binding parameters of daunorubicin and doxorubicin in the conditions used for studying the interaction of anthracycline-DNA complexes with cells in vitro. Cancer Chemother. Pharmacol. 2, 7-10 (1979).

37. Lipfert, J., Klijnhout, S. \& Dekker, N. H. Torsional sensing of small-molecule binding using magnetic tweezers. Nucleic acids Res. 38, 7122-7132 (2010).

38. Kim, Y.-J., Lee C., Lee J. G. \& Kim D.-N. J. A. N. Configurational design of mechanical perturbation for fine control of twisted DNA origami structures. ACS Nano (2019). 
39. Ke, Y. et al. Two design strategies for enhancement of multilayer-DNAorigami folding: underwinding for specific intercalator rescue and staple-break positioning. Chem. Sci. 13, 6348-6355 (2012).

40. Lee, C. et al. Tailoring the Mechanical Stiffness of DNA Nanostructures Using Engineered Defects. ACS Nano 13, 8329-8336 (2019).

41. Le Bret, M. Catastrophic variation of twist and writhing of circular DNAs with constraint? Biopolymers 18, 1709-1725 (1979).

42. Yang, Y. et al. Self-assembly of DNA rings from scaffold-free DNA tiles. Nano Lett. 13, 1862-1866 (2013).

43. Ong, L. L. et al. Programmable self-assembly of three-dimensional nanostructures from 10,000 unique components. Nature 552, 72-77 (2017)

44. Geary, C., Rothemund, P. W. \& Andersen, E. S. A single-stranded architecture for cotranscriptional folding of RNA nanostructures. Science 345, 799-804 (2014).

45. Han, D. et al. Single-stranded DNA and RNA origami. Science 358, eaao2648 (2017).

46. Sanchez, T., Kulic, I. \& Dogic, Z. Circularization, photomechanical switching, and a supercoiling transition of actin filaments. Phys. Rev. Lett. 104, 098103 (2010).

47. Praetorius, F. \& Dietz H. Self-assembly of genetically encoded DNA-protein hybrid nanoscale shapes. Science 355, eaam5488 (2017).

48. Salerno, D. et al. Magnetic tweezers measurements of the nanomechanical properties of DNA in the presence of drugs. Nucleic Acids Res. 38, 7089-7099 (2010).

49. LePecq, J.-B. \& Paoletti, C. A fluorescent complex between ethidium bromide and nucleic acids: physical-chemical characterization. J. Mol. Biol. 27, 87-106 (1967).

50. Wilner, O. I. et al. Enzyme cascades activated on topologically programmed DNA scaffolds. Nat. Nanotechnol. 4, 249-254 (2009).

51. Fu, J. et al. Multi-enzyme complexes on DNA scaffolds capable of substrate channelling with an artificial swinging arm. Nat. Nanotechnol. 9, 531 (2014).

52. Fan, S. et al. Proximity-induced pattern operations in reconfigurable DNA origami domino array. Journal of the American Chemical Society (JACS) 142, 14566-14573 (2020).

53. Douglas, S. M. et al. Rapid prototyping of 3D DNA-origami shapes with caDNAno. Nucleic Acids Res. 37, 5001-5006 (2009).

54. Lee, C., Lee, J. Y. \& Kim, D.-N. Polymorphic design of DNA origami structures through mechanical control of modular components. Nat. Commun. 8, 2067 (2017).

55. Gore, J. et al. DNA overwinds when stretched. Nature 442, 836-839 (2006).

\section{Acknowledgements}

This work was supported by the National Convergence Research of Scientific Challenges (NRF-2020M3F7A1094299) and the Basic Research Program (NRF-
2019R1A2C4069541) through the National Research Foundation of Korea Foundation of Korea (NRF) funded by Ministry of Science and ICT.

\section{Author contributions}

Y.-J.K. and D.-N.K. conceived the design approach and modeling. Y.-J.K. and J.P. performed the experiments and analyzed the data. J.Y.L. performed FE simulations and Y.-J.K. and J.Y.L. analyzed the data. Y.-J.K. and D.-N.K. discussed the results and wrote the manuscript. All authors commented on and edited the manuscript.

\section{Competing interests}

The authors declare no competing interests.

\section{Additional information}

Supplementary information The online version contains supplementary material available at https://doi.org/10.1038/s41467-021-25406-9.

Correspondence and requests for materials should be addressed to D.-N.K.

Peer review information Nature Communications thanks Maartje Bastings and the other, anonymous, reviewer(s) for their contribution to the peer review of this work. Peer reviewer reports are available.

Reprints and permission information is available at http://www.nature.com/reprints

Publisher's note Springer Nature remains neutral with regard to jurisdictional claims in published maps and institutional affiliations.

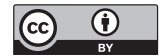

Open Access This article is licensed under a Creative Commons Attribution 4.0 International License, which permits use, sharing, adaptation, distribution and reproduction in any medium or format, as long as you give appropriate credit to the original author(s) and the source, provide a link to the Creative Commons license, and indicate if changes were made. The images or other third party material in this article are included in the article's Creative Commons license, unless indicated otherwise in a credit line to the material. If material is not included in the article's Creative Commons license and your intended use is not permitted by statutory regulation or exceeds the permitted use, you will need to obtain permission directly from the copyright holder. To view a copy of this license, visit http://creativecommons.org/ licenses/by/4.0/.

(C) The Author(s) 2021 